DOI: 10.20472/IAC.2017.031.014

\author{
ANDY CHENG \\ Hang Seng Management College, Hong Kong
}

\title{
FINANCIAL TECHNOLOGY TRANSFORMATION - EVIDENCE FROM CHINA'S VALUE WEB
}

\begin{abstract}
:
With the vigorous development in internet finance, the provisions of electronic platform service for online and mobile payments have become very popular in China. The internet finance has spurred the transformation and promoted innovation of the China's banking sector, in particular under the policies support and encouragement from the Chinese Authority. At the same time, such development also brings new challenges to the Regulators. This paper examines the current status, regulatory challenges and development of the value web in China.
\end{abstract}

Currently, internet finance in China mainly covers peer-to-peer (P2P) lending, third party online/mobile payment and online/mobile wealth management products. The value web has increased the financial inclusion and lowered the cost of entry for the public, which in return enhance user experience. With the advancement in technology, computing is getting faster and cheaper. Virtualization is driving up efficiency and utilization. Storage devices are growing in terms of capacity while declining in price. Delivery mechanism such as cloud computing also helps to lower costs and drive efficiencies. The internet finance business model is moving to Big Data application, banks expedite to draw close to both internet services and social communication network providers during the transformation process.

Information technology has brought a new competitive agent and financial intermediate. Traditionally, banks are the confluence of financial information and fund flows. However, with opening on the operations of internet companies, information gathering and integration become more efficient, extensive and faster than those from banks. While chasing economic of scales, banks in the past have concentrated their resources to those big companies (the head) which can maximize banks' profit contribution. The 'tail', those small and medium size enterprises with lower transaction amount but high frequency, is being ignored. This provides an excellent opportunity for internet finance to fill the gap. Thus, banks are facing the challenge on financial dis-intermediation during this wave of transformation.

On the other hand, crafting enlightened regulation for the internet space, in particular, in relating to financial application is never to be an easy task. It is understood that the current regulatory framework may not be able to keep abreast with the exponential growth in the value web. In view of the challenges, a comprehensive review of the Cyber Security Law in China was conducted in June 2016. Regulators recognize the importance of technological transformation of the value web and carefully adapt the approaches to regulate this new ecosystem in China.

\section{Keywords:}

Technological Change, Innovation, Mobile Business 
JEL Classification: M15, 031 